\title{
EGU21-5025
}

https://doi.org/10.5194/egusphere-egu21-5025

EGU General Assembly 2021

(c) Author(s) 2021. This work is distributed under

the Creative Commons Attribution 4.0 License.

\section{Comparison between IPSL Venus Global Climate Model results and aerobraking data}

\author{
Antoine Martinez ${ }^{1}$, Sébastien Lebonnois ${ }^{1}$, Jean-Yves Chaufray ${ }^{2}$, Ehouarn Millour ${ }^{1}$, and Thomas \\ Pierron $^{1}$ \\ ${ }^{1}$ Laboratoire de Météorologie Dynamique, Sorbonne Université, CNRS, Paris, France \\ ${ }^{2}$ LATMOS, Sorbonne Université, CNRS, Paris, France
}

For fifteen years, a Global Climate Model (GCM) has been developed for the Venus atmosphere at Institut Pierre-Simon Laplace (IPSL), in collaboration between LMD and LATMOS, from the surface up to $150 \mathrm{~km}$ altitude. Its recent extension up to the exobase (roughly $250 \mathrm{~km}$ ) within the framework of the VCD project now allows us to simulate the Venusian upper atmosphere and the key atmospheric parameters of the aerobraking phases. The aim of this presentation is to study the evolution of the density of the Venusian upper atmosphere as a function of different parameters such as solar irradiance, latitude, local time and zenith solar angle (SZA), for regions from 130 to $180 \mathrm{~km}$ of altitude. We will present here several comparisons of the upper atmosphere of Venus between our model results and a selection of aerobraking data from different missions such as Venus Express, Pioneer Venus and Magellan. 